Seyed ALI MIRNEZAMI, Eng., MSc

E-mail: mirnezamiali@gmail.com

Associate Professor Seyed MEYSAM MOUSAVI, PhD

E-mail: sm.mousavi@shahed.ac.ir (*Corresponding author)

Department of Industrial Engineering

Shahed University, Tehran, Iran

Professor Jurgita ANTUCHEVICIENE, PhD

E-mail: jurgita.antucheviciene@vgtu.It

Faculty of Civil Engineering

Vilnius Gediminas Technical University, Lithuania

Vahid MOHAGHEGHI, PhD Student

E-mail:v.mohagheghi@shahed.ac.ir

Department of Industrial Engineering

Shahed University, Tehran, Iran

\title{
A NEW APPROACH FOR MULTI-SCENARIO PROJECT CASH FLOW ANALYSIS BASED ON TODIM AND CRITICAL CHAIN METHODS UNDER GREY UNCERTAINTY
}

\begin{abstract}
Prognosticating and monitoring the financial conditions of the projects in terms of the cash are crucial to be considered for companies. Generating reliable cash flow is essential for taking proper decisions and having effective financial control. Furthermore, if the inherent uncertainties in projects are not addressed, they could lead to tremendous rates of projects failure and bankruptcy. Thus, it is crucial to consider the imprecision in projects. Therefore, the objective of this study is to anticipate the projects cash flow. In this paper, a novel approach is proposed to generate the project cash flow by applying both two extensions of critical chain project management and TODIM (an acronym in Portuguese for interactive Multi-Criteria Decision Making (MCDM)) method under grey-environments. Therefore, grey numbers are employed to state the uncertainty of activity durations, qualities, and costs. Finally, a real-world construction project is utilized to demonstrate the applicability and efficiency of proposed approach.

Keywords: Project scheduling, Cash flow, Critical chain project management, Grey theory, TODIM, MCDM.
\end{abstract}

JEL Classification: C02, C44, D81, L74, M10 
Seyed Ali Mirnezami, Seyed Meysam Mousavi, Jurgita Antucheviciene, Vahid Mohagheghi

\section{Introduction}

Since companies are vulnerable to bankruptcy, managing and planning of the projects in their financial aspects should be appropriately performed (Simion and Marin, 2018;Lucko, 2013). Although managing other resources is essential, cash is the most significant resource for companies as insufficient liquidity leads to bankruptcy (Jiang, 2011). Cash flow at the project level contains a complete history of all cash expenditures cash out flow and revenues cash inflow received from projects implementations (Park et al., 2005; Shash and Qarra, 2018). As such, creating projects cash flows during the implementation of project are not only crucial for project managers but also are important for project owners(Maravas and Pantouvakis, 2012). Therefore, preserving the balance between cash outflows and cash inflows has been a crucial matter to both prevent financial stress and ensure the smooth project accomplishment with no sufficient financing. However, preservation of the balance between cash outflows and cash inflows is complicated because of the existence of several uncertainties in projects. Meaning that, the existence of such uncertainties not just can change the fulfillment times of all activities, but also can affect on amounts of cash outflows and cash inflows directly (Mohagheghi et al., 2017; DorfeshanandMousavi, 2019; Yu et al., 2017; Bazilevych et al., 2018).

The uncertain factors, exist either in the nature of the possessions to be conveyed or in the approach of conducting it (Maravas and Pantouvakis, 2012). Besides, there are important uncertainties especially in construction projects, which come up in many aspects of the problem, consisting of the financial environments. The existing uncertainties of activity durations are due to several interdependent activities (Moradi et al., 2018; Chen et al., 2003).As such, several decisions are usually made in ambiguous circumstances and according to the vague information. Thus, the assessment of the project may turn out to be unrealistic. Even thoughthe presence of uncertainty is crucial to grasp projects, there is a shortage of a sound method for managing it. Therefore, it is essential to model and manage these uncertainties for assessing the project (Maravas and Pantouvakis, 2012).

More recently, investigations have concentrated on models to manage these uncertainties by applying stochastic, interval, fuzzy, or grey numbers (Tavanaetal., 2013; Zhang et al., 2011; Huang and Zhao, 2014; Mousavi et al., 2013, 2014). Grey system theory can be applied, as itishelpful in vague circumstances, where there is no adequate data accessible, due to its strong performances of minimum attainable data utilization (Davoudabadi et al., 2019; Ekhtiari et al., 2016; Liu and Lin, 2006).Delgado and Romero(2016) proposed a model for optimization of time, cost, and quality of the project by applying both the 
A New Approach for Multi-Scenario Project Cash Flow Analysis Based on TODIM and Critical Chain Methods under Grey Uncertainty

fuzzy goal programming and grey linear programming. Similarly, to convert qualitative information of a mining project into quantitative values, grey clustering approach was executed. Furthermore, Bhattacharyya (2015) executed the grey theory, by considering grey values for both preferences of project experts and the features ordering in portfolio selection problem. San Cristóbal et al. (2015) proposed a grey forecasting model for an anticipation of final cost of a project regarding grey system theory.

Most of the time, with respect to both preferences and criteria, activities can be performed in various ways, meaning that different scenarios can be defined for project implementation. Even though addressing such scenarios can lead to better project implementation, there is little attention paid to them. Furthermore, to the best of our knowledge, in comparison with CPM (critical path method), more investigations need to be done about CCM (critical chain management), as there is no sufficient research regarding that, in spite of the fact that it can result in less rework, time, and cost and resource constraints consideration. Thus, in this paper, different scenarios are considered for carrying out the activities, and the best scenariois selected for carrying them out based on both respective criteria and project experts' preferences; then, project scheduling is performedin accordance with the information that is conducted in the previous step and by considering the CCM method. Consequently, the respective project cash flow, which leads to better project evaluation, cost and time management, and project financing that helps to accelerate the project implementation, is conducted out of its importance not just for project managers but also for project owners. In addition to the significance of grey system theory, it is worth noting that applying such theory can lead to better consideration and management of uncertainties, enhancing project scheduling, and beingcloser to the real-world situations. Therefore, all of the values in the aforementioned steps discussed previously are considered as grey numbers.

The remainder of this study is organized by the following. An approach for conducting project cash flow based on the provided project network and adopted scenarios is presented in section 2. In section 3, a real-world project is employed to represent the applicability of the proposed algorithms. In section 4, a sensitivity analysis of essential parameters is obtained. Eventually, section 5 deduces the contributions of these investigations.

\section{Proposed methodology}

The main objective of this methodology is to generate the project cash flow. Therefore, first, different scenarios are defined; then, with respect to the criteria, the best scenario will be selected by considering TODIM method. Thus, 
Seyed Ali Mirnezami, Seyed Meysam Mousavi, Jurgita Antucheviciene, Vahid Mohagheghi

the project network will be conducted by applying CCM method, and after that in accordance with this project network, the project cash flow will be obtained. Moreover, to manage the uncertainties in the project, all of the values are considered as grey numbers.

\subsection{Phase 1: Scenario evaluation}

Most of the time, when implementing activities of a project, there are different ways of achieving them. In other words, there are different scenarios to implement them. Therefore, by examining both the criteria, such as time, cost, and quality and the information about these criteria; the project managers have to decide on the best scenario and its implementation so that results in optimization and improvement of the criteria. It is necessary to determine how to decide on the best scenario for each activity among the others. In this way, to the involvement of the different criteria, scenarios, and project experts in decision-making, the TODIM method will be executed. Thus, the best scenario for the implementation of an activity can be selected using this method. This method is a new grey extension on a classical TODIM method, which is first presented by Gomes (2009), and its steps are as follows:

Step 1. Initially, a set of $(n)$ scenarios for each activity with respect to $(m)$ quantitative and qualitative criteria is considered to be orderedandconsequently, the best scenario will be chosen among the others. Thus, both scenarios and criteria are defined. After defining the respective component, project experts are asked to evaluate each of the qualitative criteria $(c)$, it is necessary to convert the qualitative criteria estimated on a verbal scale into a numerical scale. Furthermore, by considering the performance of the scenarios concerning the criteria, the estimations of the quantitative criteria will be conducted. Hence, by evaluating the scenarios in respect of all criteria by the project experts concerning both definition of existence scenario, quantitative, and qualitative criteria respectively; the matrix of evaluation $(D)$ will be generated.

$\otimes D=\left[\begin{array}{cccc}\otimes G_{11} & \otimes G_{12} & \ldots & \otimes G_{1 m} \\ \otimes G_{21} & \bigotimes G_{22} & \ldots & \otimes G_{2 m} \\ \vdots & & \ddots & \vdots \\ \otimes G_{n 1} & \bigotimes G_{n 2} & \ldots & \otimes G_{n m}\end{array}\right]$

where $\otimes D$ is the grey decision matrix and $\otimes G_{i j}$ is the grey rating of $i$ th scenario with respect to $j$ th criterion. 
A New Approach for Multi-Scenario Project Cash Flow Analysis Based on TODIM and Critical Chain Methods under Grey Uncertainty

Step 2. Hereinafter, it is essential that the normalized performances of the matrix are considered. Hence, the normalized grey decision matrix $\left(\otimes D^{*}\right)$ will be as follows.

$\otimes D^{*}=\left[\begin{array}{cccc}\otimes G^{*}{ }_{11} & \bigotimes G^{*}{ }_{12} & \ldots & \otimes G^{*}{ }{ }^{*} \\ \otimes G^{*}{ }_{21} & \bigotimes G^{*}{ }_{22} & \ldots & \otimes G^{*}{ }^{2 m} \\ \vdots & & \ddots & \vdots \\ \otimes G^{*}{ }_{n 1} & \bigotimes G^{*}{ }_{n 2} & \ldots & \bigotimes G^{*}{ }_{n m}\end{array}\right]$

where $\otimes G^{*}{ }_{i j}$ is the normalized grey rating of $i$ th scenario with respect to $j$ th criterion that is expressed as:

$\otimes G^{*}{ }_{i j}=\frac{\otimes G i j}{\sum_{i=1}^{n} \otimes G i j}$

Step 3. After the definition of each criterion, their respective weights will be assigned regarding the importance of criteria that is carried out by experts through the evaluation on a cardinal scale. As well as the previous step, performing the normalization for each criterion is essential to be considered herein. In this way, the assigned weight of each criterionis divided by the sum assigned weights of all the criteria. Then, one of the criterion $(r)$ will be selected as a reference criterion among the others according to its importance for calculations by project experts. Thus, the reference criterion will usually be a criterion with the most influence, which is assigned to it. Concerning the calculation of the reference criterion and the normalized weights on a cardinal scale that are assigned to each criterion by decision-makers; $\left(w_{r c}\right)$, which result in translating the differences between performance values to the same dimension, will be conducted using the division of the weight of criterion $(c)$ to the weight of the reference criterion $(r)$. A measurement of a dominance of each scenario $\left(A_{i}\right)$ over each scenario $\left(A_{j}\right)$ will be conducted by the following:

$\delta\left(A_{i}, A_{j}\right)=\sum_{c=1}^{m} \Phi_{c}\left(A_{i}, A_{j}\right) \forall(i, j)$

where:

DOI: $10.24818 / 18423264 / 54.2 .20 .16$ 
Seyed Ali Mirnezami, Seyed Meysam Mousavi, Jurgita Antucheviciene, Vahid Mohagheghi

$$
\Phi_{c}\left(A_{i}, A_{j}\right)= \begin{cases}\sqrt{\frac{w_{r c}}{\sum_{c=1}^{m} w_{r c}} \cdot d\left(\otimes G_{i c}, \otimes G_{j c}\right)} & \text { if }\left[\otimes G_{i c}>\otimes G_{j c}\right] \\ 0, & \text { if }\left[\otimes G_{i c}=\otimes G_{j c}\right] \\ -\frac{1}{\theta} \sqrt{\frac{\sum_{c=1}^{m} w_{r c}}{w_{r c}} \cdot d\left(\otimes G_{i c}, \otimes G_{j c}\right)} & \text { if }\left[\otimes G_{i c}<\otimes G_{j c}\right]\end{cases}
$$

$d\left(\otimes G_{i c}, \otimes G_{j c}\right)$ in both Eq. (5) and Eq. (6) denotes theEuclidean distance.

If $\otimes G_{1}=\left[\underline{G_{1}}, \overline{G_{1}}\right]$ and $\otimes G_{2}=\left[\underline{G_{2}}, \overline{G_{2}}\right]$ are two grey number set then, Euclidean distance between two grey numbers $\otimes G_{1}$ and $\otimes G_{2}$ can be calculated by using the below equation:

$$
d\left(\otimes G_{1}, \otimes G_{2}\right)=\sqrt{\frac{1}{2}\left[\left(\underline{G_{1}}-\underline{G_{2}}\right)^{2}+\left(\overline{G_{1}}-\overline{G_{2}}\right)^{2}\right]}
$$

where $\delta\left(A_{i}, A_{j}\right)$ indicates the measurement of the dominance of scenario $A_{i}$ over scenario $A_{j} ; m$ is the number of criteria; $c$ is any criterion, for $c=1, \ldots, m ; w_{c}$ is the weight of criterion $c ; w_{r}$ is the weight of the reference criterion; $r$ is the reference criterion; $\otimes G_{i c}$ and $\otimes G_{j c}$ are the normalized grey rating against criterion $c$ for two scenarios $A_{i}$ and $A_{j}$, respectively; $d\left(\otimes G_{i c}, \otimes G_{j c}\right)$ designates the distance between the two grey numbers $\otimes G_{i c}$ and $\otimes G_{j c}$, as defined in Equation ( 8 ); $\theta$ is the attenuation factor of the losses; $\Phi_{c}\left(A_{i}, A_{j}\right)$ represents the parcel of the contribution of criterion $c$ to function $\delta\left(A_{i}, A_{j}\right)$, when comparing scenario $i$ with scenario $j$.

When calculating $\Phi_{c}\left(A_{i}, A_{j}\right)$, if $\left[\otimes G_{i c}>\otimes G_{j c}\right]$, Eq. (5) will be used, If $\left[\otimes G_{i c}=\right.$ $\left.\otimes G_{j c}\right]$, the value zero will be assigned to $\Phi_{c}\left(A_{i}, A_{j}\right)$, meaning that, Eq. (6) will be applied, and If $\left[\otimes G_{i c}<\otimes G_{j c}\right]$, Eq. ( 7 ) will be executed. After calculating $\Phi_{c}\left(A_{i}, A_{j}\right)$ for each criterion, through the sum of its components, $\delta\left(A_{i}, A_{j}\right)$ will be obtained consequently, which is represented by Eq. (4). 
A New Approach for Multi-Scenario Project Cash Flow Analysis Based on TODIM and Critical Chain Methods under Grey Uncertainty

Step 4. Ultimately, to conduct the overall value of scenario $I$ through normalization of the corresponding dominance measurements, the following expression is executed:

$$
\xi_{i}=\frac{\sum_{j=1}^{n} \delta\left(A_{i}, A_{j}\right)-\min \sum_{j=1}^{n} \delta\left(A_{i}, A_{j}\right)}{\max \sum_{j=1}^{n} \delta\left(A_{i}, A_{j}\right)-\min \sum_{j=1}^{n} \delta\left(A_{i}, A_{j}\right)}
$$

where $\xi_{i}$ provides the rank of each scenario. From the above, it is concluded that the overall values of the scenarios are calculated as a result of the normalization. Building upon these, the rank ordering of all scenarios, regarding the ordering of the respective values will be obtained eventually. Thus, the best scenario can be selected among the others consequently.

\subsection{Phase 2:Grey CCM}

Compared to critical path method in which activities start as soon as possible, the primary objective of CCPM (critical chain project management), which was first introduced by Goldratt (1997), is reaching a project network in which activities start as late as possible. It is supposed that as a result of its implementation the projects can be carried out with less time and cost in comparison with other methods.The grey CCM method is described in this paper based on Leach (2014). Moreover, the calculation is conducted by the following steps.

Step 5. Initially, the activities are scheduled by considering the predecessors and the estimated duration of activities. After identifying the required resources in CCPM, called "resource loading", the CPM plan ought to be resourceleveled.

$\otimes E S_{\text {istart }}=[\underline{0}, \overline{0}]$

$$
\otimes E S_{i}=\max \left[\otimes E F_{p}\right]
$$

$\otimes E F_{i}=\otimes E S_{i}+\otimes D_{i}$

$\otimes E F_{i}=\left[\underline{e s_{i}}, \overline{e s_{i}}\right]+\left[\underline{d_{i}}, \overline{d i}\right]$

where $\otimes E S_{i}=\left[\underline{e s_{i}}, \overline{e s_{i}}\right], \otimes E F_{i}=\left[\underline{e f_{i}}, \overline{e f_{i}}\right]$, and $\otimes D_{i}=\left[\underline{d_{i}}, \overline{d i}\right]$ are activities with an early start, early finish, and duration, respectively. 
Seyed Ali Mirnezami, Seyed Meysam Mousavi, Jurgita Antucheviciene, Vahid Mohagheghi

Step 6. Most of the time, there is an amount of safety time when estimating the duration of activities by using the $C P M$ method that, according to the $C C P M$ method, is useless and leads to prolongation of projects. Thus, to eliminate the safety time, which is estimated by experts, one-half of the estimated duration for each activity $\otimes D_{\text {old } i}=\left(\underline{d_{\text {old } i}}, \overline{d_{\text {old } i}}\right)$ is considered to calculate the final duration $\left(\otimes D_{\text {new } i}\right)$.

if $\otimes D_{\text {old } i}=\left(\underline{d_{\text {old } i}}, \overline{d_{\text {old } i}}\right)$ then $\otimes D_{\text {new } i}=\frac{1}{2}\left(\otimes D_{\text {old } i}\right)$

Step 7. Initially, the critical chain that can be defined as a set of both precedence and resource-dependent activities that, if delayed, will extend the end date of the project; then, feeding chain, which is the set of activities that tieinto one of the activity that is placed in the critical chain, are determined. Afterward, unlike $C P M$ in which activities start as soon as possible, in $C C M$ activities begin as late as possible, meaning that activitiesare scheduled in terms of the end date of projects.

$$
\begin{gathered}
\otimes L F_{i}=\min \left[\otimes L S_{p}\right] \\
\otimes L S_{i}=\otimes L F_{i}-\otimes D_{i} \\
\otimes L S_{i}=\left[\underline{l f_{i}}, \overline{l f_{i}}\right]-\left[\underline{d_{i}}, \overline{d_{i}}\right]
\end{gathered}
$$

where $\otimes L S_{i}=\left[\underline{l s_{i}}, \overline{l s_{i}}\right], \otimes L F_{i}=\left[l \underline{f_{i}}, \overline{l f_{i}}\right]$, and $\otimes D_{i}=\left[\underline{d_{i}}, \overline{d i}\right]$ are activities with a late start, late finish, and duration, respectively.

Step 8. It has been a pressing matter how to size the project buffer properly; therefore, the project buffer which is considered to be equal to one-half of the resulting critical chain is calculated and added to the end of the critical chain as follows:

$$
\begin{gathered}
\otimes B=\frac{1}{2}\left[\sum_{\substack{i=1 \\
\forall i \in k}}^{n} \otimes D_{i}\right] \\
\otimes E S_{B} \geq \otimes E S_{j}+\otimes D_{j}
\end{gathered}
$$

where $\otimes B$ indicates the project buffer; $\otimes D_{i}=\left[\underline{d_{i}}, \overline{d i}\right]$ is the duration of activities; $k$ is a set of activities that are in critical chain; $n$ is the number of activities; $\otimes E S_{B}$ is the early start of project buffer; $\otimes E S_{j}$ and $\otimes D_{j}$ are early start and duration of 
A New Approach for Multi-Scenario Project Cash Flow Analysis Based on TODIM and Critical Chain Methods under Grey Uncertainty

activity $j$, which is the last activity of the critical chain and predecessor of the project buffer.

Step 9. It is considered that the method, which is applied by experts to size the project buffer and the process utilized to size feeding buffer are alike. Thus, feeding buffer will be equal to one-half of the feeding chain, and it is placed at the point, the last activity on feeding chain tie into the successor on the critical chain.

$$
\begin{gathered}
\otimes F B=\frac{1}{2}\left[\sum_{\substack{i=1 \\
\forall i \in F C}}^{n} \otimes D_{i}\right] \\
\otimes E S_{F B} \geq \otimes E S_{j}+\otimes D_{j}
\end{gathered}
$$

where $\otimes F B$ indicates the feeding buffer; $\otimes D_{i}=\left[\underline{d_{i}}, \overline{d i}\right]$ is the duration of activities; $F C$ is a set of activities that are in feeding chain; $n$ is the number of activities; $\otimes E S_{F B}$ is the early start of feeding buffer; $\otimes E S_{j}$ and $\otimes D_{j}$ are early start and duration of activity $j$, which is the last activity of feeding chain and predecessor of the feeding buffer.

\subsection{Phase 3: Cash flow analysis}

Financing during the project can lead to an acceleration of the project implementation. As such, it is believed that accurate cash flow is urgent for evaluating the projects, monitoring and controlling the projects, and ascertaining the project financing requirements. Thus, the creation of project cash flow is not only crucial for project managers but also significant for project owners. To calculate the project cash flow, the cost distribution per unit of time needs to be summarized for the whole project. The calculation is conducted by the following steps, which are a grey extension of the method utilized by Maravas and Pantouvakis (2012).

Step 10. Owing to the different duration of activities,two types of optimistic and pessimistic situations are defined for the best case $\operatorname{Min} \otimes D_{i}$, which means the activity starts as soon as possible and lasts the minimum duration, and for the worst-case $\operatorname{Max} \otimes D_{i}$, which expresses the activity starts as late as possible and lasts the maximum duration. It is supposed that $\otimes E S_{i}=\left[e s_{i}, \overline{e s_{i}}\right], \otimes E F_{i}=$ $\left[e f_{i}, \overline{e f_{i}}\right]$, and $\otimes D_{i}=\left[\underline{d_{i}}, \overline{d i}\right]$ are activities with an early start, early finish, and duration respectively. The intervals of these two cases are calculated as follows: 
Seyed Ali Mirnezami, Seyed Meysam Mousavi, Jurgita Antucheviciene, Vahid Mohagheghi

$\operatorname{Min} \otimes D_{i}=\left[e s_{i}, e s_{i}+d_{i}\right]$

$\operatorname{Max} \otimes D_{i}=\left[\overline{e s_{i}}, \overline{e s_{i}}+\overline{d i}\right]$

Step 11. The maximum and the minimum cost distribution per unit of time can be calculated by dividing the cost of every activity by both the optimistic and the pessimistic cases of the activity duration respectively, which is denoted as:

$\otimes C_{i}=\left[\underline{c_{i}}, \overline{c_{i}}\right]$, For Activity $A: \otimes C_{A}=\left[\underline{c_{A}}, \overline{c_{A}}\right]$

$\otimes C_{i_{\min }}=\left[\underline{c_{i}}, \overline{c_{i}}\right]$

$\operatorname{Min} \otimes C D_{i t}=\frac{\left[\underline{c_{i}}, \overline{c_{i}}\right]}{\left[\underline{e s_{i}}, \underline{e s_{i}}+\underline{d_{i}}\right]}$

$\otimes C_{i_{\max }}=\left[\underline{c_{i}}, \overline{c_{i}}\right]$

$\operatorname{Max} \otimes C D_{i t}=\frac{\left[\underline{c_{i}}, \overline{c_{i}}\right]}{\left[\overline{e s_{i}}, \overline{e s_{i}}+\overline{d i}\right]}$

where $\otimes C_{i}=\left[\underline{c_{i}}, \overline{c_{i}}\right]$ indicates the cost of every activity; $\otimes C_{A}=\left[\underline{c_{A}}, \overline{c_{A}}\right]$ is the cost of activity $A ; \operatorname{Max} \otimes C D_{i}$ and $\operatorname{Min} \otimes C D_{i}$ are the maximum and the minimum cost distribution per unit of time; $\left[\underline{e s_{i}}, \underline{e s_{i}}+\underline{d_{i}}\right]$ and $\left[\overline{e s_{i}}, \overline{e s_{i}}+\overline{d i}\right]$ are both the optimistic and the pessimistic cases of the activity duration, respectively.

Step 12. The total cost $\otimes C_{t}$ at a specific time period $t$ for the whole project is equal to the sum of the cost distribution per unit of time $t$ from each activity $i=1$ to $n$.

$\operatorname{Min} \otimes C_{t}=\sum_{i=1}^{n} \operatorname{Min} \otimes C D_{i t}$

$\operatorname{Max} \otimes C_{t}=\sum_{i=1}^{n} \operatorname{Max} \otimes C D_{i t}$

Step 13. Eventually, the project cost curve (cash flow - CF) is the cumulative plot of the cost per time period for the project duration $(T)$. In other words, project cash flow is equal to the sum of the total cost per time period for the project duration, which is calculated as follows:

$\operatorname{Min} \otimes C F_{t}=\sum_{t=0}^{T} \operatorname{Min} \otimes C_{t}$ 
A New Approach for Multi-Scenario Project Cash Flow Analysis Based on TODIM and Critical Chain Methods under Grey Uncertainty

$\operatorname{Max} \otimes C F_{t}=\sum_{t=0}^{T} \operatorname{Max} \otimes C_{t}$

Furthermore, concerning the two cases which are defined hereinbefore, there are two min and max cumulative plots of cost. As such, the cash flow uncertainty $(C F U)$ is defined by the following:

$$
C F U=\operatorname{Max} \otimes C F_{t}-\operatorname{Min} \otimes C F_{t}
$$

\section{Case study}

To demonstrate the applicability of the proposed model, a real-world project is presented in the oil and gas industry. It is planned for a Petro-Refinery Complex (PRC) situated in Bushehr Province in Iran to produce petrochemical products. It was prognosticated for an incipient design of the South Pars Gas Field Development Project. This project not only comprised the products like light and sweet gas for the national gas and liquefied natural gas pipelines, but also it was predicted for the project to produce other products like ethane, propane, butane, and pentane from the gas that has delivered to national gas company.To produce these products as mentioned above, the company works on several projects. One of these projects, which consists of six main activities, is considered in this paper. All data, which is collected in this case study, is byPetro refining project reports.

In the first phase, the objective is to select the best scenario for the implementation of activities. In this way, all respective data is shown in Table 1. In the first and second columns, activities with dependent relationships are displayed. Since there are different ways for implementation of activities, depending on how to implement an activity, different durations, costs, and qualities are considered for each of them, which are shown in third, fourth, and fifth columns, respectively. Contrary to the two first criteria, named duration and cost respectively, which are quantitative, the last criterion, named quality, is qualitative, and its scale can be observed in Table 2 as associated grey score.

Table 1.Activity network data

\begin{tabular}{|c|c|c|c|c|c|c|c|c|c|c|}
\hline \multirow{2}{*}{ Activities } & \multirow{2}{*}{ Predecessors } & \multicolumn{3}{|c|}{ First scenario } & \multicolumn{3}{|c|}{ Second scenario } & \multicolumn{3}{|c|}{ Third scenario } \\
\hline & & 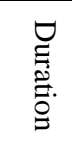 & $\begin{array}{l}2 \\
\infty\end{array}$ & 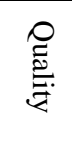 & $\begin{array}{l}\underset{\Xi}{\Xi} \\
\text { 苛. } \\
\stackrel{0}{\Xi}\end{array}$ & $\begin{array}{l}2 \\
0\end{array}$ & 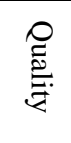 & $\begin{array}{l}Ð \\
\Xi \\
\stackrel{\Xi}{0} . \\
\stackrel{0}{g}\end{array}$ & $\begin{array}{l}0 \\
\wp\end{array}$ & 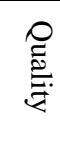 \\
\hline $\bar{A}$ & $\begin{array}{l}---- \\
\end{array}$ & $\begin{array}{l}45, \\
70]\end{array}$ & $\begin{array}{c}4500, \\
6000]\end{array}$ & $\mathrm{MG}$ & $\begin{array}{l}45, \\
70]\end{array}$ & $\begin{array}{c}4500, \\
6000]\end{array}$ & $\overline{\mathrm{MG}}$ & $\begin{array}{l}45, \\
70]\end{array}$ & $\begin{array}{c}{[4500,} \\
6000]\end{array}$ & $\overline{\mathrm{MG}}$ \\
\hline $\mathrm{B}$ & A & $\begin{array}{c}{[35,} \\
55]\end{array}$ & $\begin{array}{l}{[3500,} \\
4500]\end{array}$ & M & $\begin{array}{c}{[30,} \\
50]\end{array}$ & $\begin{array}{l}{[3000,} \\
4000]\end{array}$ & $\mathrm{G}$ & $\begin{array}{c}{[40,} \\
60]\end{array}$ & $\begin{array}{c}{[4000,} \\
5000]\end{array}$ & VG \\
\hline
\end{tabular}


Seyed Ali Mirnezami, Seyed Meysam Mousavi, Jurgita Antucheviciene, Vahid Mohagheghi

\begin{tabular}{ccccccccccc}
\hline $\mathrm{C}$ & $\mathrm{A}$ & {$[30$,} & {$[1800$,} & $\mathrm{G}$ & {$[30$,} & {$[1800$,} & $\mathrm{G}$ & {$[30$,} & {$[1800$,} & $\mathrm{G}$ \\
& & $50]$ & $2500]$ & & $50]$ & $2500]$ & & $50]$ & $2500]$ & \\
$\mathrm{D}$ & $\mathrm{B}, \mathrm{C}, \mathrm{F}$ & {$[80$,} & {$[2000$,} & $\mathrm{G}$ & {$[90$,} & {$[2200$,} & $\mathrm{VG}$ & {$[85$,} & {$[2500$,} & $\mathrm{M}$ \\
& & 100 & $3000]$ & & 120 & $3400]$ & & 110 & $3500]$ & \\
& & ] & & & ] & & & ] & & \\
$\mathrm{E}$ & $\mathrm{D}$ & {$[55$,} & {$[420,6$} & $\mathrm{VG}$ & {$[50$,} & {$[450,6$} & $\mathrm{M}$ & {$[45$,} & {$[400,6$} & $\mathrm{G}$ \\
& & $80]$ & $30]$ & & $75]$ & $50]$ & & $70]$ & $00]$ & \\
$\mathrm{F}$ & $\mathrm{A}$ & {$[20$,} & {$[2500$,} & $\mathrm{MG}$ & {$[20$,} & {$[2500$,} & $\mathrm{MG}$ & {$[20$,} & {$[2500$,} & $\mathrm{MG}$ \\
& & $40]$ & $3000]$ & & $40]$ & $3000]$ & & $40]$ & $3000]$ & \\
\hline
\end{tabular}

Table 2. Valuation of criterion $C_{3}$ - execution quality

\begin{tabular}{cc}
\hline Execution quality & Associated grey score \\
\hline Very poor (VP) & {$[0,2]$} \\
Poor (P) & {$[2,4]$} \\
Medium poor (MP) & {$[4,5]$} \\
Medium (M) & {$[5,6]$} \\
Medium good (MG) & {$[6,7]$} \\
Good (G) & {$[7,8]$} \\
Very good (VG) & {$[8,9]$} \\
\hline
\end{tabular}

Moreover, Table 3 presents both final values of the scenarios obtained through normalization after carrying out the mathematical computations of the TODIM method and ordering of each scenario. Consequently, $B_{2}, D_{1}$, and $E_{3}$ are the scenarios that are chosen for the activities in Table 3, respectively.

Table 3. Final values and ordering

\begin{tabular}{cccc}
\hline Activities & Scenarios & Normalized global values & Ordering \\
\hline \multirow{2}{*}{$B$} & $B_{1}$ & {$[-457.1,217.8]$} & 2 \\
& $B_{2}$ & {$[-457.5,457.5]$} & 1 \\
& $B_{3}$ & {$[-488.5,1]$} & 3 \\
$D$ & $D_{1}$ & {$[-33.24,33.24]$} & 1 \\
& $D_{2}$ & {$[-35,25,1]$} & 3 \\
& $D_{3}$ & {$[-33.57,16.16]$} & 2 \\
$E$ & $E_{1}$ & {$[-50.2,1]$} & 3 \\
& $E_{2}$ & {$[-48.04,19.61]$} & 2 \\
& $E_{3}$ & {$[-47.82,47.82]$} & 1 \\
\hline
\end{tabular}

In the second phase, by considering the results obtained through the lastphase, project scheduling is conducted by applying the CCM method to compute both early start time and early finish time activities. Table 4 denotes these results. 
A New Approach for Multi-Scenario Project Cash Flow Analysis Based on TODIM and Critical Chain Methods under Grey Uncertainty

Table 4. Grey-project scheduling

\begin{tabular}{ccc}
\hline Activities & Early start & Early finish \\
\hline A & {$[0,0]$} & {$[22.5,35]$} \\
B & {$[22.5,35]$} & {$[37.5,60]$} \\
C & {$[37.5,60]$} & {$[52.5,85]$} \\
Feeding buffer & {$[47.5,75]$} & {$[52.5,85]$} \\
D & {$[52.5,85]$} & {$[92.5,135]$} \\
E & {$[92.5,135]$} & {$[115,170]$} \\
F & {$[37.5,55]$} & {$[47.5,75]$} \\
Project buffer & {$[115,170]$} & {$[172.5,255]$} \\
\hline
\end{tabular}

According to Table 4, a grey two-dimensional Gantt chart schedule with the project early start and finish dates, which are presented by grey-numbers, is illustrated in Figure 1 in which $x$-axis indicates time, the $y$-axis shows the activity name. Unlike the ordinary Gantt charts, activity durations determined by grey numbers - the grey start date and the grey completion date-which results in the real start and completion activity boundaries.

Eventually, in the third phase, the project cash flow is calculated based on the cost of activities and also early start time and early finish time activities. With respect to two types of optimistic and pessimistic situations, first, the cost distribution per unit of time is obtained. Then, based on cost distribution, project cash flow, which is equal to the sum of the total cost per period for the project duration, is calculated. The results for optimistic and pessimistic situations are 14200 and 19098.9, respectively.

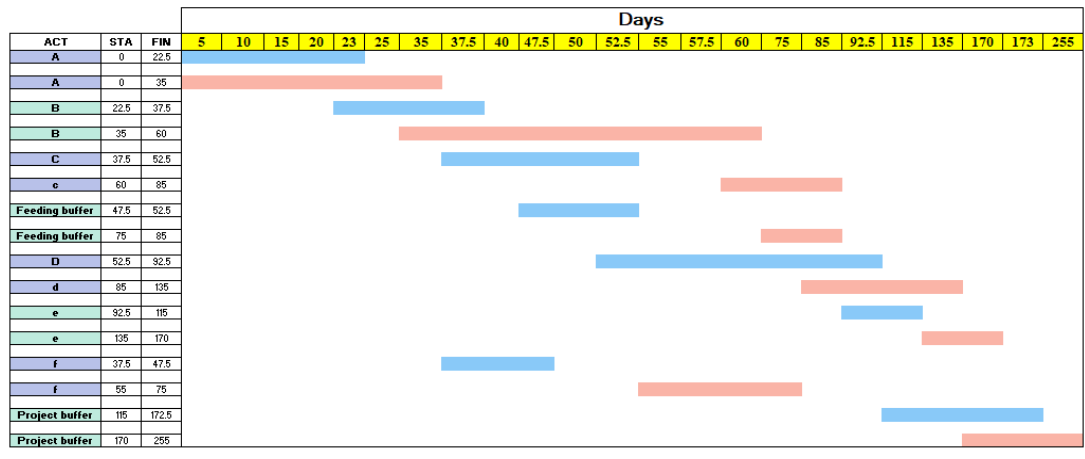

Figure 1. Grey-Gantt chart 
Seyed Ali Mirnezami, Seyed Meysam Mousavi, Jurgita Antucheviciene, Vahid Mohagheghi

\section{Sensitivity analysis}

Moreover, for the purpose of assessing outcomes of the proposed approach, a sensitivity analysis is performed by considering different criteria's weights for three respective activities, in which its results are presented in Table 5.

Table 5. Sensitivity analysis on criteria' weights

\begin{tabular}{|c|c|c|c|c|c|c|c|}
\hline \multirow[t]{2}{*}{ Activities } & \multirow[t]{2}{*}{ Conditions } & \multicolumn{3}{|c|}{ Weights } & \multicolumn{3}{|c|}{$\begin{array}{l}\text { Scenario } \\
\text { ranking }\end{array}$} \\
\hline & & $C_{1}$ & $C_{2}$ & $C_{3}$ & $G_{1}$ & $G_{2}$ & $G_{3}$ \\
\hline \multirow{4}{*}{ B } & main & {$[0.001,0.33]$} & {$[0.16,0.66]$} & {$[0.33,0.99]$} & 2 & 1 & 3 \\
\hline & 1 & {$[0.001,0.33]$} & {$[0.33,0.99]$} & {$[0.16,0.66]$} & 2 & 3 & 1 \\
\hline & 2 & {$[0.16,0.66]$} & {$[0.001,0.33]$} & {$[0.33,0.99]$} & 2 & 1 & 3 \\
\hline & 3 & {$[0.33,0.99]$} & {$[0.16,0.66]$} & {$[0.001,0.33]$} & 1 & 2 & 3 \\
\hline \multirow{3}{*}{$\mathrm{D}$} & main & {$[0.001,0.33]$} & {$[0.16,0.66]$} & {$[0.33,0.99]$} & 1 & 3 & 2 \\
\hline & 1 & {$[0.001,0.33]$} & {$[0.33,0.99]$} & {$[0.16,0.66]$} & 1 & 3 & 2 \\
\hline & 2 & {$[0.16,0.66]$} & {$[0.001,0.33]$} & {$[0.33,0.99]$} & 2 & 1 & 3 \\
\hline \multirow{5}{*}{$\mathrm{E}$} & 3 & {$[0.33,0.99]$} & {$[0.16,0.66]$} & {$[0.001,0.33]$} & 2 & 3 & 1 \\
\hline & main & {$[0.001,0.33]$} & {$[0.16,0.66]$} & {$[0.33,0.99]$} & 3 & 2 & 1 \\
\hline & 1 & {$[0.001,0.33]$} & {$[0.33,0.99]$} & {$[0.16,0.66]$} & 3 & 2 & 1 \\
\hline & 2 & {$[0.16,0.66]$} & {$[0.001,0.33]$} & {$[0.33,0.99]$} & 2 & 3 & 1 \\
\hline & 3 & {$[0.33,0.99]$} & {$[0.16,0.66]$} & {$[0.001,0.33]$} & 3 & 1 & 2 \\
\hline
\end{tabular}

The object of the analysis is to exchange each criterion's weight with another one. Consequently, three combinations of three criteriaare assessed for three activities, with each combination stated as a condition. The main condition indicates the original outcomes of the case study. In accordance with Table 6, $G_{3}, G_{2}$, and $G_{1}$ have the highest rank for activity B, when the first, second, and third criteria weights are exchanged in conditions 1, 2, and 3 respectively. However, when the same exchange of criteria weights is performed for activity $\mathrm{D}, G_{1}, G_{2}$, and $G_{3}$ have the highest rank in conditions 1,2, and 3, respectively. Besides, $G_{3}$ has the highest rank in both condition 1, and condition 2 for activity E. contrary to the two first conditions for activity $\mathrm{E}$ in which $G_{3}$ was the best scenario, $G_{2}$ has the highest rank in the third one. Thus, $G_{3}$ has the highest rank in all conditions other than the third one in this activity. Therefore, with respect to the above-mentioned table, for instance, it is obvious that choosing $G_{3}$ and $G_{1}$ for activities $\mathrm{E}$ and $\mathrm{D}$ as the best scenarios of activities implementation for the Petro refining project is more reliable.

\section{Conclusions}

In comparison with the lack of profit in the projects, insufficient cash has a higher influence on companies going bankrupt due to its importance for supporting progressing project activities. Thus, forecasting accurate project cash flow is 
A New Approach for Multi-Scenario Project Cash Flow Analysis Based on TODIM and Critical Chain Methods under Grey Uncertainty

extremely crucial not only for project managers but also for contractors. Moreover, due to the risky nature of projects, there are significant uncertainties, especially in construction projects, which lead to the invalidity of project evaluations. Thus, it is necessary to manage them for project assessment. Therefore, a sound approach including activities with grey durations, qualities, and costs is introduced in this paper, aiming at providing project cash flow management under uncertain environment by considering both critical chain project management for early start and finish time calculation and TODIM method to select the best scenario. The proposed approach, initially adopted the best scenario, which indicated the best way of activities execution, by conducting TODIM method under greyenvironment regarding project experts' preferences and criteria. Then, early start time and early finish time of activities were calculated by applying a grey extension of critical chain project management, in which the projects were implemented considering resource constraints with less time and cost in comparison with CPM. Subsequently, the project cash flow considering uncertainties was generated with respect toproject network for both evaluating the projects and providing project managers with a comprehensive insight into uncertainties and project financing requirements. Ultimately, a real-world project was applied to demonstrate the assessment methods application. Therefore, in the case study, the best scenario for execution of each activity was adopted. Then, the grey-early start time and early finish time were calculated,and the respective Gantt chart was illustrated. To compare this method with the critical path method, the same data was considered for both of them. Without considering the resource constraints, the total project duration, determined by critical path method was $(200,290)$ days. When the resource leveling was conducted, the total project duration was extended to $(230,340)$ days. By using this method, a project schedule with a $(172.5,255)$-day project duration was obtained. Then, the project cash flow was generatedand after that, outcomes of the case study were discussed. Finally, sensitivity analysis of significant parameters was achieved.

\section{REFERENCES}

[1] Bazilevych, K., Mazorchuk, M., Parfeniuk, Y., Dobriak, V., Meniailov, I., \&Chumachenko, D. (2018), Stochastic Modelling of Cash Flow for Personal Insurance Fund Using the Cloud Data Storage.International Journal of Computing, 17(3), 153-162;

[2] Bhattacharyya, R. (2015), A Grey Theory Based Multiple Attribute Approach for $\boldsymbol{R} \& D$ Project Portfolio Selection. Fuzzy Information and Engineering, 7(2), 211-225; 
Seyed Ali Mirnezami, Seyed Meysam Mousavi, Jurgita Antucheviciene, Vahid Mohagheghi

[3] Chen, C. H., Ling, S. F. \& Chen, W. (2003), Project Scheduling for Collaborative Product Development Using DSM.International Journal of Project Management, 21(4), 291-299;

[4] Davoudabadi, R., Mousavi, S.M., Šaparauskas, J.\&Gitinavard H. (2019),Solving Construction Project Selection Problem by a New Uncertain Weighting and Ranking Based on Compromise Solution with Linear Assignment Approach; Journal of Civil Engineering and Management, 25(3), 241-251;

[5] Delgado, A. \& Romero, I. (2016), Environmental Conflict Analysis Using an Integrated Grey Clustering and Entropy-weight Method: A Case Study of a Mining Project in Peru.Environmental Modelling \& Software, 77, 108-121;

[6] Dorfeshan, Y.\&Mousavi, S.M. (2019), A Group TOPSIS-COPRAS Methodology with Pythagorean Fuzzy Sets Considering Weights of Experts for Project Critical Path Problem;Journal of Intelligent and Fuzzy Systems, 36(2), 1375-1387;

[7] Ekhtiari, M., Yadegari, E. \&Sadidi, G. (2016), Ranking Entrepreneurship Main Risks in Non-Profit Financial Funds by TODIM Technique under Grey Conditions (A Case Study in IRAN).Economic Computation \& Economic Cybernetics Studies \& Research, 50(3); ASE Publishing;

[8] Gomes, L. F. A. M. (2009), An Application of the TODIM Method to the Multicriteria Rental Evaluation of Residential Properties. European Journal of Operational Research, 193(1), 204-211;

[9] Huang, X. \& Zhao, T. (2014), Project Selection and Scheduling with Uncertain Net Income and Investment Cost.Applied Mathematics and Computation, 247, 61-71;

[10] Jiang, A., Issa, R. R. \&Malek, M. (2011), Construction Project Cash Flow Planning Using the Pareto Optimality Efficiency Network Model.Journal of Civil Engineering and Management, 17(4), 510-519;

[11] Leach, L. P. (2014), Critical Chain Project Management.Artech House;

[12] Liu, S. \& Lin, Y. (2006), Grey Information: Theory and Practical Applications. Springer Science \& Business Media;

[13] Lucko, G. (2013), Supporting Financial Decision-Making Based on Time Value of Money with Singularity Functions in Cash Flow Models.Construction Management and Economics, 31(3), 238-253;

[14] Maravas, A. \&Pantouvakis, J. P. (2012), Project Cash Flow Analysis in the Presence of Uncertainty in Activity Duration and Cost. International Journal of Project Management, 30(3), 374-384;

[15] Mohagheghi, V., Mousavi, S. M. \&Vahdani, B. (2017), Analyzing Project Cash Flow by a New Interval Type-2 Fuzzy Model with an Application to Construction Industry. Neural Computing and Applications, 28(11), 33933411 
A New Approach for Multi-Scenario Project Cash Flow Analysis Based on TODIM and Critical Chain Methods under Grey Uncertainty

[16] Mousavi, S.M., Jolai, F., Tavakkoli-Moghaddam, R.\&Vahdani, B. (2013), A Fuzzy Grey Model Based on the Compromise Ranking for MultiCriteria Group Decision Making Problems in Manufacturing Systems;Journal of Intelligent and Fuzzy Systems, 24(4), 819-827;

[17] Mousavi, S.M., Vahdani, B., Tavakkoli-Moghaddam, R.\& Tajik, N. (2014),Soft Computing Based on a Fuzzy Grey Compromise Solution Approach with an Application to the Selection Problem of Material Handling Equipment; International Journal of Computer Integrated Manufacturing, 27(6), 547-569;

[18] Moradi, N., Mousavi, S. M. \&Vahdani, B. (2018), An Interval Type-2 Fuzzy Model for Project-earned Value Analysis under Uncertainty. Journal of Multiple-Valued Logic \& Soft Computing, 30(1), 79-103;

[19] Park, H. K., Han, S. H. \& Russell, J. S. (2005), Cash Flow Forecasting Model for General Contractors Using Moving Weights of Cost Categories.Journal of Management in Engineering, 21(4), 164-172;

[20] San Cristóbal, J. R., Correa, F., González, M. A., de Navamuel, E. D. R., Madariaga, E., Ortega, A. \&Trueba, M. (2015), A Residual Grey Prediction Model for Predicting S-Curves in Projects. Procedia Computer Science, 64, 586-593;

[21] Shash, A. A. \&Qarra, A. A. (2018), Cash Flow Management of Construction Projects in Saudi Arabia.Project Management Journal, 49(5), 48-63;

[22] Simion, C. P. \& Marin, I. (2018), Project Cost Estimate at Completion: Earned Value Management versus Earned Schedule-Based Regression Models. A Comparative Analysis of the Models Application in the Construction Projects in Romania. Economic Computation \& Economic Cybernetics Studies \& Research, 52(3); ASE Publishing;

[23] Tavana, M., Khalili-Damghani, K. \&Sadi-Nezhad, S. (2013), A Fuzzy Group Data Envelopment Analysis Model for High-Technology Project Selection: A Case Study at NASA. Computers \& Industrial Engineering, 66(1), 10-23;

[24] Yu, M. C., Dang, V. L. \&Yeh, H. C. (2017), Measuring Cash Flow and Overdraft for Fuzzy Project Networks with Overlapping Activities.Journal of Civil Engineering and Management, 23(4), 487-498;

[25] Zhang, W. G., Mei, Q., Lu, Q. \& Xiao, W. L. (2011), Evaluating Methods of Investment Project and Optimizing Models of Portfolio Selection in Fuzzy Uncertainty. Computers \& Industrial Engineering, 61(3), 721-728. 